\title{
A Cas12a ortholog with stringent PAM recognition followed by low off-target editing rates for genome editing
}

Peng Chen ${ }^{1 \dagger}$, Jin Zhou ${ }^{1 \dagger}$, Yibin Wan ${ }^{1 \dagger}$, Huan Liu', Yongzheng Li ${ }^{1}$, Zhaoxin Liu' ${ }^{1}$, Hongjian Wang ${ }^{1}$, Jun Lei Kai Zhao ${ }^{1}$, Yiliang Zhang ${ }^{3}$, Yan Wang ${ }^{3}$, Xinghua Zhang ${ }^{4}$ and Lei Yin ${ }^{1 *}$

\begin{abstract}
Background: AsCas12a and LbCas12a nucleases are reported to be promising tools for genome engineering with protospacer adjacent motif (PAM) TTV as the optimal. However, the C-containing PAM (CTV, TCTV, TTCV, etc.) recognition by Cas12a might induce extra off-target edits at these non-canonical PAM sites.

Results: Here, we identify a novel Cas12a nuclease CeCas12a from Coprococcus eutactus, which is a programmable nuclease with genome-editing efficiencies comparable to AsCas12a and LbCas12a in human cells. Moreover, CeCas12a is revealed to be more stringent for PAM recognition in vitro and in vivo followed by very low off-target editing rates in cells. Notably, CeCas12a renders less off-target edits located at C-containing PAM at multiple sites compared to LbCas12a and AsCas12a, as assessed by targeted sequencing methods.

Conclusions: Our study shows that CeCas12a nuclease is active in human cells and the stringency of PAM recognition could be an important factor shaping off-target editing in gene editing. Thus, CeCas12a provides a promising candidate with distinctive characteristics for research and therapeutic applications.
\end{abstract}

Keywords: CRISPR, Cas12a, Gene editing, PAM stringency, Off-targeting

\section{Background}

Clustered regularly interspaced short palindromic repeats (CRISPR)/CRISPR-associated nucleases (Cas), which are essential for bacterial adaptive immunity, have been exploited to develop potent tools for genome manipulation in cells and organisms and have widespread applications in research, medicine, and biotechnology [1-10]. Besides the most commonly used Streptococcus pyogenes Cas9 (SpCas9), a series of Cas9 orthologs from different organisms, such as Staphylococcus aureus (Sa), Streptococcus thermophilus (St), and Neisseria meningitidis (Nm),

\footnotetext{
* Correspondence: yinlei@whu.edu.cn

†Peng Chen, Jin Zhou and Yibin Wan contributed equally to this work.

'State Key Laboratory of Virology, Hubei Key Laboratory of Cell Homeostasis, Department of Biochemistry and Molecular Biology, College of Life Sciences, Wuhan University, Wuhan, China

Full list of author information is available at the end of the article
}

have been exploited [11-13]. However, SpCas9 presents unsurpassed activity in gene editing and genome manipulation.

Recently, CRISPR-Cas12a/Cpf1 was reported to be a highly specific programmable nuclease with high efficiency comparable to Cas9 [14-16]. Several different features make Cas12a an important expansion of CRISPRbased genome-editing tools [14]. First, Cas12a needs only a single crRNA processed to be mature by itself without the requirement of a trans-activating RNA (tracrRNA) and double-stranded (ds) RNA-specific ribonuclease RNase III, which are indispensable for maturation of Cas9 crRNA. Second, Cas12a requires thymine-rich protospacer adjacent motif (PAM) sequence at the $5^{\prime}$ end of the protospacer, different from the guanine-rich PAM sequences at the 3' end of the target DNA for Cas9 systems. Third,

(c) The Author(s). 2020 Open Access This article is licensed under a Creative Commons Attribution 4.0 International License, which permits use, sharing, adaptation, distribution and reproduction in any medium or format, as long as you give appropriate credit to the original author(s) and the source, provide a link to the Creative Commons licence, and indicate if changes were made. The images or other third party material in this article are included in the article's Creative Commons licence, unless indicated otherwise in a credit line to the material. If material is not included in the article's Creative Commons licence and your intended use is not permitted by statutory regulation or exceeds the permitted use, you will need to obtain permission directly from the copyright holder. To view a copy of this licence, visit http://creativecommons.org/licenses/by/4.0/ The Creative Commons Public Domain Dedication waiver (http://creativecommons.org/publicdomain/zero/1.0/) applies to the data made available in this article, unless otherwise stated in a credit line to the data. 
after cleavage of double-stranded DNAs, Cas12a generates a staggered double-strand break resulting in 4 or $5 \mathrm{nt} 5^{\prime}$ overhangs distal to the PAM site; however, Cas9 creates blunt ends within the PAM-proximal target site. Due to the features discussed above, Cas12a can be an alternative even better tool for genome editing in some situations. Moreover, it was suggested that Cas12a is highly specific in human cells with low off-target rates from the study of two types of Cas12a (Acidaminococcus sp. BV3L6, AsCas12a, and Lachnospiraceae bacterium ND 2006, LbCas12a) $[17,18]$.

These distinct features of Cas12a provide good potential for the development of genomic editing tools. However, some challenges may still hinder the application of CRISPRCas systems, such as how some certain factors shape offtargeting and how to reduce that. We noticed off-target effects at non-canonical PAM region from the previous study of Feng Zhang and his colleagues which adopted engineered Cas12a variants to alter PAM specificities for increasing genome targeting range [19]. From this clue, a novel Cas12a nuclease from Coprococcus eutactus (CeCas12a) was identified to be more restrictive in the selection of PAM sequences in vitro and in vivo than AsCas12a and LbCas12a, followed by the lower off-targeting in cells at the PAM region, and the off-targeting of CeCas12a at other regions was also lower in general. Besides, the efficiency of CeCas12a in editing human cells was comparable to well-accepted AsCas12a and LbCas12a. All these suggested CeCas12a could be a good expansion of accurate CRISPR-based genome-editing tools and stringency for PAM recognition might be an important factor shaping off-targeting which could lead us to find more useful accurate genome-editing tools.

\section{Results}

Cas12a orthologs with different stringencies for recognizing canonical TTTV and non-canonical Ccontaining PAMs in vitro

Cas12a (Cpf1) nucleases such as AsCas12a, LbCas12a, and FnCas12a have been used as gene-editing tools in biological researches with the requirement of recognition of specific PAM sequences, and non-canonical PAM regions were also recognized in some extent [14, 15, 20]. From previous others' data, we noticed off-target effects could happen at non-canonical PAM regions [19, 21]. To further investigate the stringency for recognizing non-canonical PAMs and the related off-target effects at non-canonical PAMs region by different Cas12a orthologs, 12 Cas12a nucleases were selected including four Cas12a nucleases (CeCas12a, PrCas12a from Prevotella ruminicola strain BPI-34, CsbCas12a from Candidatus Saccharibacteria bacterium, BhCas12 from Butyrivibrio hungatei strain MB2003) which have not previously been reported, 3 Cas12a nucleases (AsCas12a, LbCas12a, FnCas12a) with activity for genome manipulation, and another 5 reported Cas12a nucleases (SsCas12a from Smithella sp. SC_K08D17, Lb3Cas12a from Lachnospiraceae bacterium MC2017, BpCas12a from Bytyrivibrio, PdCas12a from Prevotella disens, BfCas12a from Butyrivibrio fibrisolvens MD2001) which have not been employed for genome editing on endogenous sites in human cells (Additional file 1: Figure S1A) [14, 22]. Their crRNA arrays were identified from their genomic loci (Additional file 2: Table S1). Two hundred fifty-six kinds of linear dsDNA substrates were synthesized consisting of 4 randomized nucleotides upstream of the protospacer with overlap PCR (Additional file 1: Figure S3A). Then, the Cas12a nucleases expressed in Escherichia coli cells were purified (Additional file 1: Figure S2A, B) and incubated with in vitro-transcribed crRNAs and dsDNA substrates for in vitro DNA cleavage assays (Additional file 1: Figure S3B). Except for AsCas12a, LbCas12a, and FnCas12a, which had been reported to be with DNA cleavage activity in vitro and in vivo, only CeCas12a and BfCas12a were able to cleave dsDNA in vitro and were chosen for further investigations (Additional file 1: Figure S4, 5, 6, 7). CeCas12a and BfCas12a could recognize $5^{\prime}$-TTTN PAM to cleave the linear dsDNA substrates (Fig. 1a; Additional file 1: Figure S4A, B), which is similar to AsCas12a and LbCas12a [14]. The DNA cleavage activities of LbCas12a and AsCas12a have been investigated both in vitro $[14,23]$ and in vivo $[17$, $18,24]$. Similarly, with the purpose to check the characters of these Cas12a orthologs for genome editing, we measured dsDNA cleavage activities of all these Cas12a orthologs in vitro using linear dsDNA substrates with 23-nt target sequences and the TTTA PAM (Additional file 1: Figure S8A). CeCas12a and BfCas12a present robust DNA cleavage activity in vitro (Additional file 1: Figure S8A). To further determine the $\mathrm{PAM}$ of $\mathrm{Ce}$ and $\mathrm{Bf}$, we adapted an in vitro PAM identification assay. We incubated Cas12a RNP complex and 150-bp dsDNA substrates containing 4 randomized nucleotides upstream of the same target spacer. By amplifying and deep sequencing the remaining DNA substrates and comparing with the negative control, we found that CeCas12a and BfCas12a also recognize rich T PAMs (Fig. 1b, d). We also detected whether the fourth nucleotide of the PAM could influence the cleavage activities of Cas12a orthologs; CeCas12a and BfCas12a nucleases showed similar cleavage activities. Both efficiently cleaved the TTTA, TTTG, and TTTC sites, but slightly cleaved the TTTT site (Fig. 1a). Although Cas12a orthologs recognized the TTTV as the optimal PAM, AsCas12a and Lb Cas12a also recognized C-containing PAMs, such as CTTV, TCTV, and TTCV (V is A, G, or C) [23, 25].

Both CeCas12a and BfCas12a recognized the TTTV PAM and C-containing PAMs (Fig. 1a, c, d; Additional file 1: Figure S4A, B). However, for CeCas12a, the 


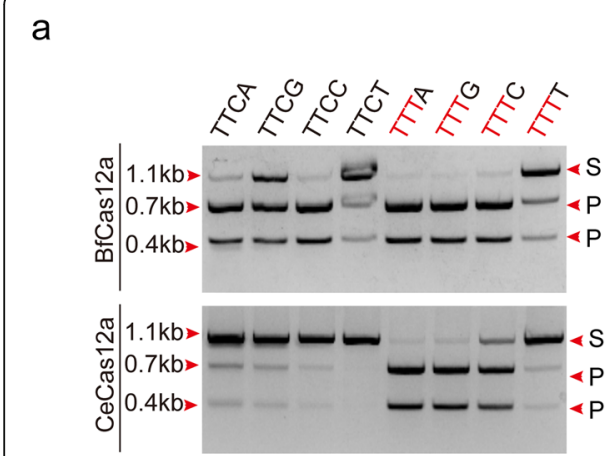

C

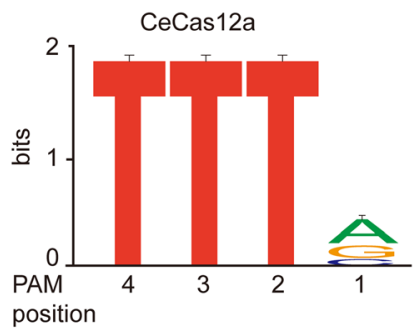

b

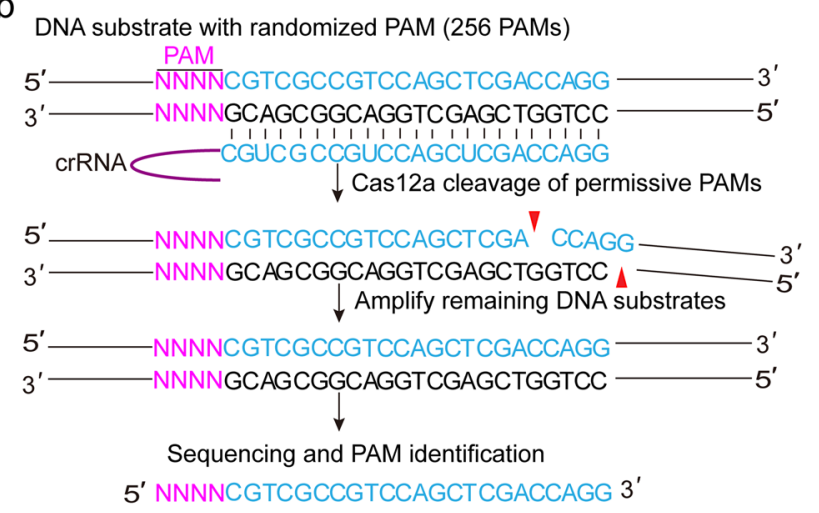

PAM position $\quad 4321$

d

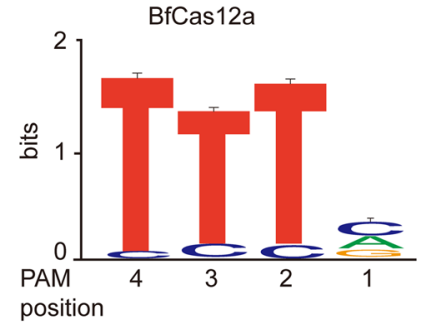

e

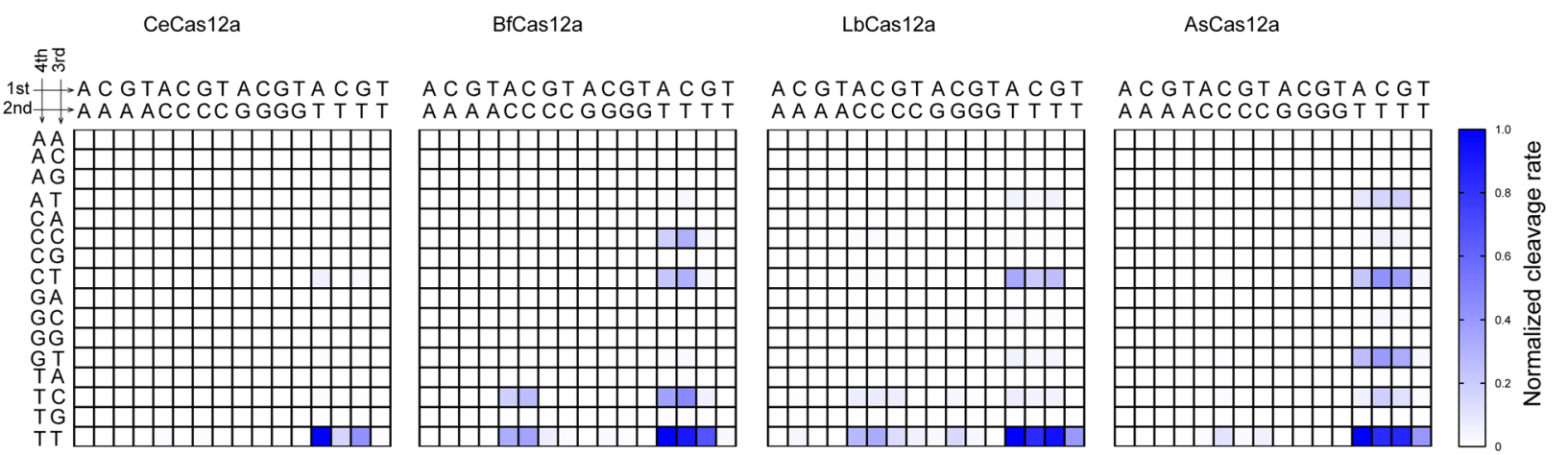

f
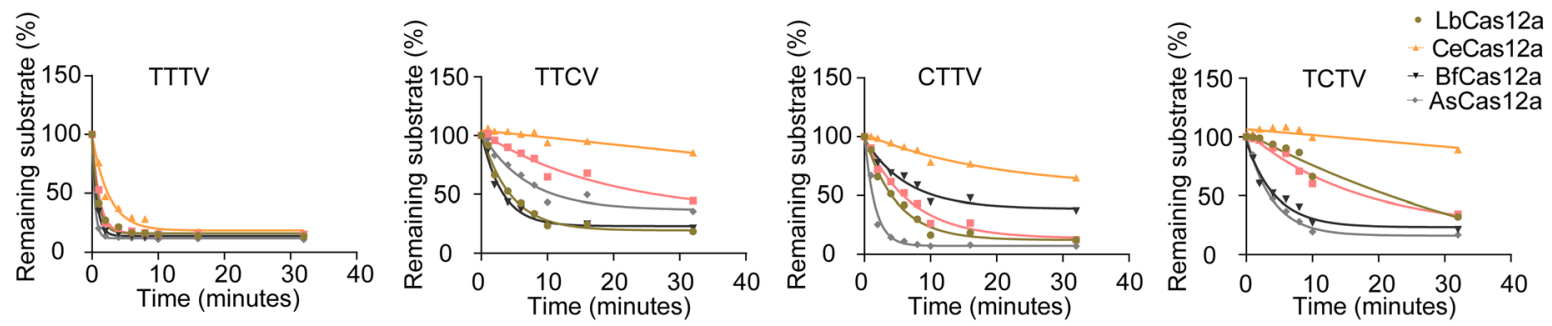

Fig. 1 DNA cleavage of Cas12a orthologs in vitro. a DNA cleavage activities of BfCas12a and Cas12a in vitro. The Cas12a-crRNAcomplex (100 nM) was incubated at $37^{\circ} \mathrm{C}$ for $10 \mathrm{~min}$ with DNA substrates (see also Additional file 1: Figure S4). b Schematic of in vitro cleavage assay used to identify PAM sequence and determine global PAM specificity. $\mathbf{c}$ Web logo for the CeCas12a PAM. $\mathbf{d}$ Web logo for the BfCas12a PAM. e Normalized cleavage rates for all 4base PAMs for CeCas12a, BfCas12a, LbCas12a, and AsCas12a. The intensity of color represented the activity of Cas12a nuclease. $\mathbf{f}$ Quantification of time-course in vitro cleavage reactions of Cas12a orthologs on linearized dsDNA substrates. Those were conducted at nine time points, respectively. Curves were fit using the one phase exponential decay equation 
suboptimal C-containing PAM [25] substrates were slightly cleaved (such as CTTV and TTCV PAMs) or even barely cleaved (such as TCTV and CCTV PAMs) while the optimal PAM TTTV substrates were cleaved completely (Additional file 1: Figure S4A). To assess the PAM preference of CeCas12a and BfCas12a and compare them with two widely used Cas $12 \mathrm{a}$ nucleases (AsCas12a, LbCas12a), we systematically studied reactions in parallel for Cas12a nucleases by incubating for a different amount of time for each candidate to assess cleavage kinetics (Fig. 1e, f and Additional file 3). Normalized cleavage rate reaches maximum at TTTV PAM (Fig. 1e), and it supports TTTV as the optimal PAM for CeCas12a, BfCas12a, AsCas12a, and LbCas12a. Certain PAMs could also be recognized by Cas12a nucleases as suboptimal candidates (Fig. 1e). In detail, AsCas12a, LbCas12a, and BfCas12a cleaved other dsDNA substrates including TCTV, TTCV, and CTTV at detectable rates (Fig. 1e, f). CeCas12a had the comparable cleavage rate to AsCas12a and LbCas12a toward TTTV PAM (Fig. 1e, f). However, CeCas12a had a lower cleavage rate at CTTV and nearly undetectable rates at TCTV and TTCV, compared to AsCas12a and LbCas12a at those PAMs (Fig. 1e, f). Their cleavage activities in vitro were also systematically measured at the same target site with 7 different C-containing PAMs (TCTA, TTCA, TCCA, CTTA, CTCA, CCTA, and CCCA) (Additional file 1: Figure S8B). Five Cas12a nucleases show different cleavage activities at the same PAM site. For the optimal TTTA site, all Cas12a nucleases present robust DNA cleavage activities and nearly all substrates were cleaved (Additional file 1: Figure S8B). Although C-containing PAM substrates could be cleaved by all five Cas12a nucleases, the efficacies were lower than TTTA PAM substrate (Additional file 1: Figure S8B). Especially for CeCas12a, there were few cleavages at $\mathrm{C}$-containing PAM sites except the CTTA PAM site (Additional file 1: Figure S8B). To further confirm their specificities or stringency for different PAMs, we incubated each Cas12a RNP and dsDNA substrates containing TTTV, TCTV, TTCV, and CTTV (V = A, G, and C) in parallel at nine different amount of time $(0 \mathrm{~min}, 1 \mathrm{~min}, 2 \mathrm{~min}, 4$ min, $6 \mathrm{~min}, 8 \mathrm{~min}, 10 \mathrm{~min}, 16 \mathrm{~min}, 32 \mathrm{~min}$ ), by deep sequencing the remaining DNA substrates and comparing them with the negative control, in order to assess cleavage kinetics (Additional file 1: Figure S8C). As expected, all Cas12a nucleases were most active at TTTV $(\mathrm{V}=\mathrm{A}$, $\mathrm{G}$, and $\mathrm{C}$ ) and Ce presented little activities at those $\mathrm{C}$ containing PAMs.

All these results showed that AsCas12a and LbCas12a recognized TTTV and non-canonical C-containing PAMs in vitro, which were consistent with previous studies [23, 25]. Furthermore, another two novel Cas12a nucleases (BfCas12a and CeCas12a) were identified with comparable cleavage efficiencies to AsCas12a, LbCas12a, and FnCas12a in vitro. Among them, CeCas12a was the least active at TCTA, TTCA, and CTTA PAMs, even barely active with some PAMs such as TCCA, CTCA, and CCTA (Fig. 1e, f; Additional file 1: Figure S8B, C). CeCas12a turned out to be more specific on PAM recognition in vitro, which drove us to further investigate whether this could shape off-targeting in cells.

Gene editing with CeCas12a and BfCas12a in human cells Cas12a can be programmed to induce DNA doublestrand breaks (DSBs) at specific genomic loci in vivo [14, $15,17,18,20,25,26]$. To test if CeCas12a and BfCas12a could be harnessed for gene editing in mammalian cells, we optimized the codons of two genes and attached a Cterminal and an $\mathrm{N}$-terminal nuclear localization signals (NLS) for optimal expression and nuclear targeting in human cells. They were then cloned into mammalian expression vector pcDNA3.1. In order to monitor the activity of CeCas12a and BfCas12a, a series of crRNAs with different lengths of spacers (13 nt, $15 \mathrm{nt}, 17 \mathrm{nt}, 19$ $\mathrm{nt}, 21 \mathrm{nt}, 23 \mathrm{nt}, 25 \mathrm{nt}, 27 \mathrm{nt}, 29 \mathrm{nt}, 31 \mathrm{nt})$ targeting the same site of EGFP were cloned into pU6-Fn-crRNA vector (Addgene number \#78958) which was driven by a U6 promoter. To detect cleavage efficiency of CeCas12a and BfCas12a nucleases, we adopted an EGFP disruption assay [27-29] that was based on generations of frameshift indel mutations, thus leading to the loss of fluorescence. Significant EGFP disruption was observed for crRNAs with 19-25 nt spacer sequences, which are commonly used for Cas12a, and over 60\% EGFP disruption efficiency for CeCas12a (Fig. 2a) and 40\% for BfCas12a were achieved (Fig. 2b). To provide appropriate controls, we also identified two inactivated RuvC nuclease domain mutants for CeCas12a (D880A, E975A) and BfCas12a (D834A, E925A) nucleases, based on the sequence similarity of these nucleases [14, 30] (Fig. 2a, b; Additional file 1: Figure S9A, B, C, D). To further confirm the in vivo activity, the cleavage assay was refined by using $23 \mathrm{nt}$ spacer crRNA targeting the same site of EGFP with six Cas12a nucleases (AsCas12a, LbCas12a, FnCas12a, PcCas12a, PdCas12a, and cMtCas12a) which had been reported before [14]. The results also showed that CeCas12a and BfCas12a displayed robust EGFP disruption activities when pairing an EGFP-targeted crRNA (Fig. 2c; Additional file 1: Figure S10).

Previous studies suggested that the crRNA carrying nucleotide substitutions in the direct repeats had variable effects on the cleavage activity of Cas12a nucleases $[15,31]$. To investigate the effects of mutations in the loop region, we employed 8 crRNA loop regions (AsCas12a, 5-UCUU3; LbCas12a, 5-UAAGU-3; FnCas12a/BfCas12a/CeCas12a, 5-UGUU-3; PbCas12a/PeCas12a/LiCas12a, 5-UUUU-3; Lb2Cas12a/PmCas12a/PcCas12a, 5-UAUU; MbCas12a, 5- 

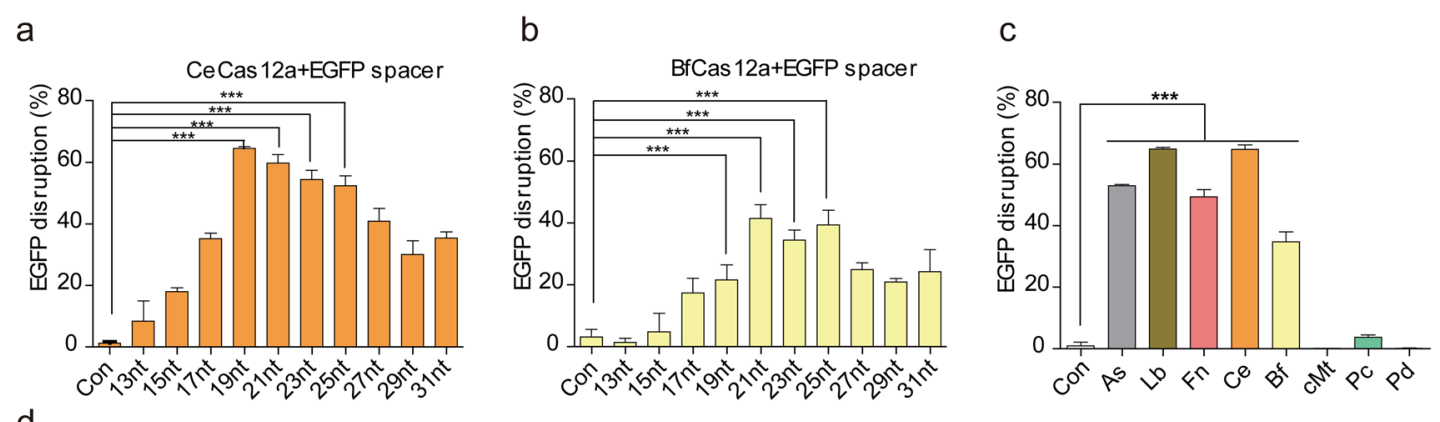

d
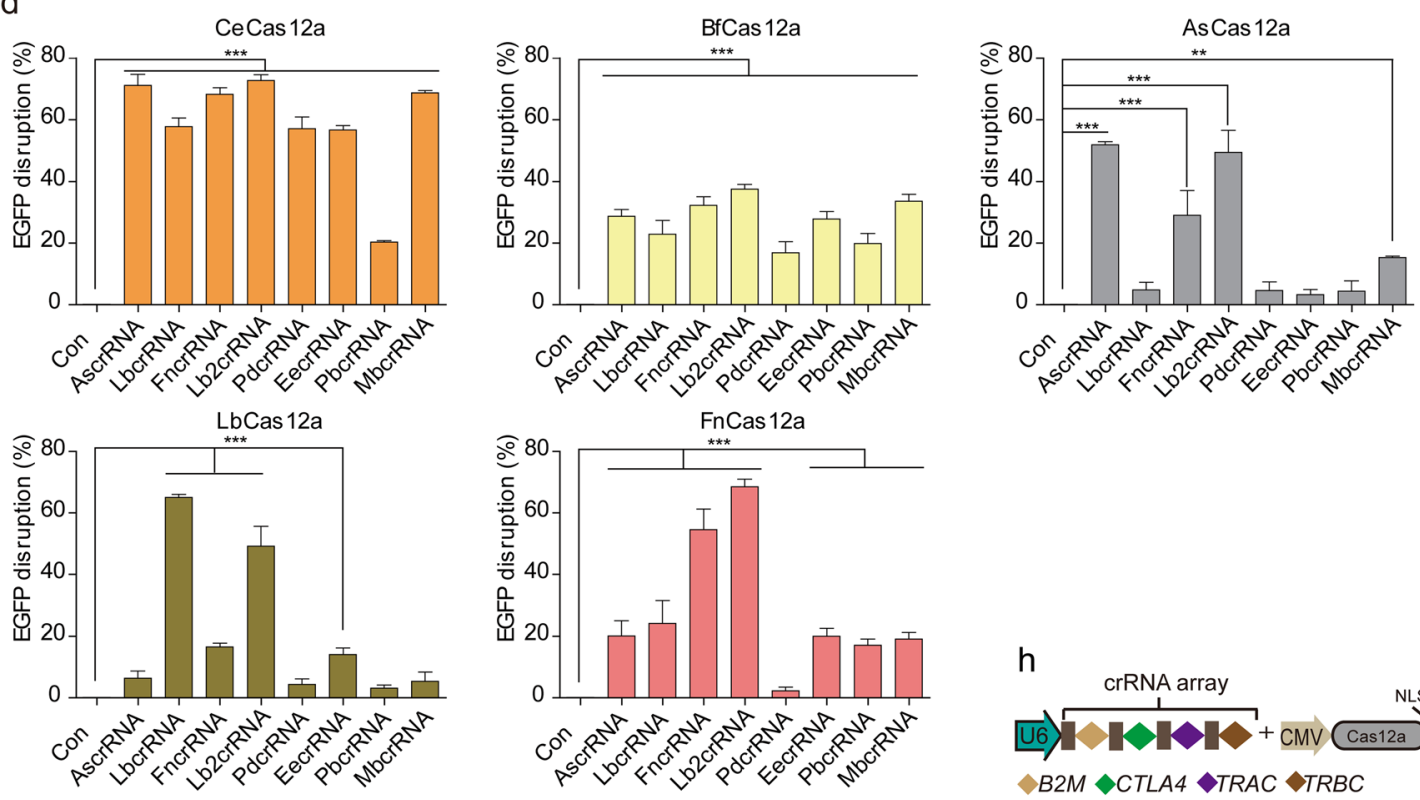

e

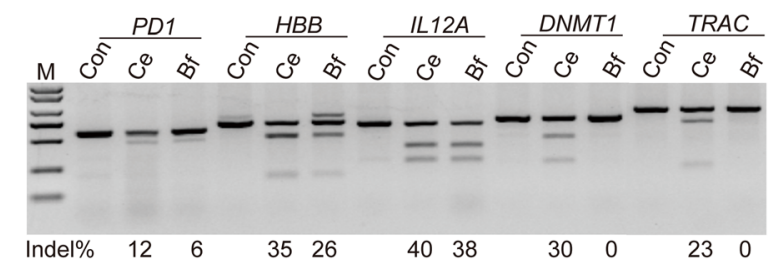

f

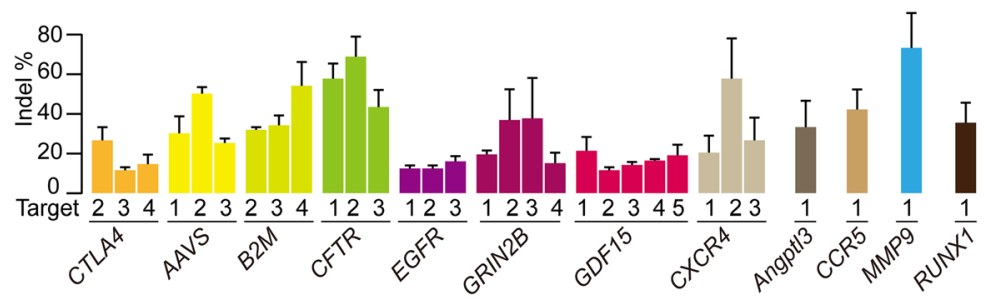

h crRNA array

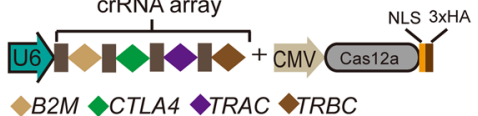

$\triangle B 2 M \triangle C T L A 4-T R A C \rightarrow T R B C$
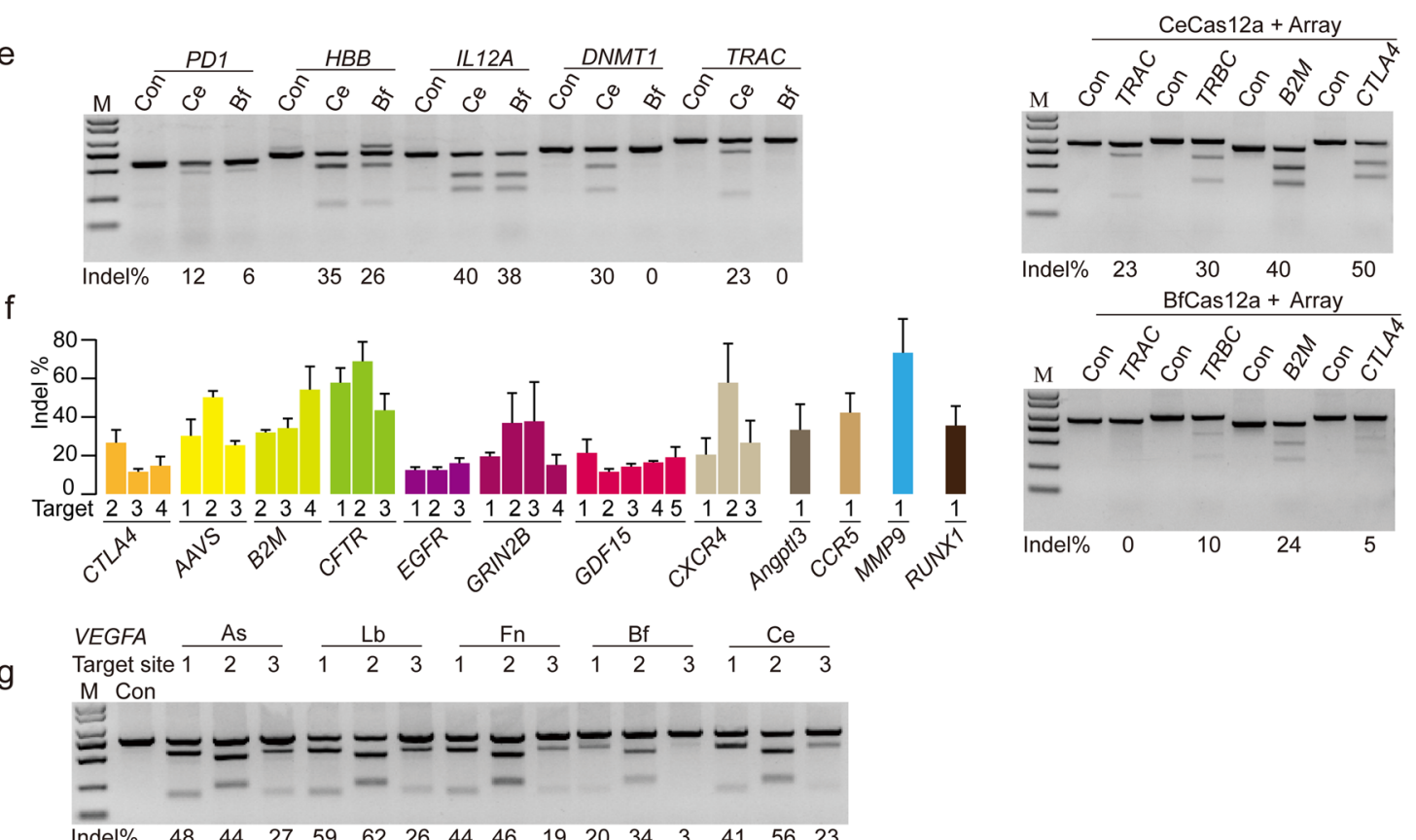

Fig. 2 (See legend on next page.) 
(See figure on previous page.)

Fig. 2 CeCas12a and BfCas12a mediate gene editing in human cells. a Efficiencies of EGFP disruption in human cells mediated by CeCas12a and crRNAs bearing variable-length complementarity regions for the target site of EGFP in human cells. Error bars indicate standard errors of means (s.e.m.), $n=3$. ${ }^{* *} P<0.001$ (Mann-Whitney). $\mathbf{b}$ Efficiencies of EGFP disruption mediated by BfCas12a and crRNAs bearing variable-length complementarity regions for the target site of EGFP in human cells. Error bars represent s.e.m., $n=3$. ${ }^{* *} P<0.001$ (Mann-Whitney). c Efficiencies of EGFP disruption in human cells mediated by Cas12a orthologs and crRNA bearing 23-nt length complementarity regions for the target site of EGFP in human cells. Error bars represent s.e.m., $n=3$. ${ }^{* *} P<0.001$ (Mann-Whitney). $\mathbf{d}$ The suitability of Cas12a with crRNAs containing different loop regions from Cas12a orthologs. Data are shown as mean \pm s.e.m. $(n=3)$; ${ }^{*} P<0.01,{ }^{* *} P<0.001$ (Mann-Whitney). e Efficiencies of targeted indel mutations introduced at five different human endogenous gene targets by CeCas12a and BfCas12a. Indel frequencies of PD1, HBB, IL12A, DNMT1, and TRAC were measured by T7E1 assay. f Summaries of the activities of CeCas12a at TTTV PAMs at a diverse panel of target sites in HEK293T cells. For indel percentages, each column represents the mean of $n=3$ transfected cell cultures. Indel frequencies were analyzed by T7E1 assay. $\mathbf{g}$ Comparison of CeCas12a and Cas12a orthologs' gene-editing efficiencies. Three target sites are from VEGFA loci. Indel frequencies analyzed by T7E1 assay. $\mathbf{h}$ Cas12a-mediated multiplex gene editing in human cells. Schematic of CRISPR array construct with the U6 direct repeat spacer cassette containing B2M, CTLA4, TRAC, and TRBC protospacer sequences (top). Indel frequencies were analyzed by T7E1 assay after transfection with CRISPR array and Cas12a plasmids (bottom)

UGUUU-3; PdCas12a, 5-UUCG-3; EeCas12a, 5-UUU-3) from 14 Cas12a orthologs and combined these crRNA members with different Cas12a nucleases (Additional file 1: Figure S1B). The EGFP disruption ratio analysis by FACS revealed that the highest cleavage efficiency was achieved by the Cas12a nuclease with its cognate crRNA loop region in most cases (Fig. 2d). Furthermore, all five Cas12a nucleases can utilize crRNA spacer with Lb2crRNA loop region to cleave target DNA which is consistent with the previous studies of $\mathrm{As} / \mathrm{Lb} / \mathrm{Fn}$ Cas12a nucleases $[15,18$, 32] (Fig. 2d). Among them, CeCas12a could nearly accommodate all different crRNA loop regions to achieve impressive cleavage efficiencies (Fig. 2d).

We further explored the capability of CeCas12a and BfCas12a to cleave different genomic loci ( $H B B, D N M T 1$, IL12A, TRAC, and PD1) in human cells and quantified gene-editing efficiencies (Fig. 2e). The results of the T7E1 assay showed that CeCas12a and BfCas12a could generate DNA double-strand breaks in human 293T cells. Deep sequencing results further confirmed the ability of CeCas12a and BfCas12a to introduce insertions or deletions (indels) at target sites in mammalian genomes (Additional file 1: Figure S11). In some cases, BfCas12a almost lost the potential for gene editing. In contrast, CeCas12a could perform pretty well in all 5 genomic targets (Fig. 2e). Furthermore, we detected another 31 endogenous target sites located on CTLA4, B2M, AAVS, CFTR, EGFR, GRIN2B, GDF15, CXCR4, Angptl3, CCR5, MMP9, and RUNX1, and the mean frequencies of indels range from 8 to $65 \%$ (Fig. 2f; Additional file 1: Figure S12). For evaluating the gene-editing potential of CeCas12a and BfCas12a along with well-accepted $\mathrm{As} / \mathrm{Lb} / \mathrm{Fn}$ Cas12a nucleases, three target sites of VEGFA were checked. All three sites could be successfully cleaved in human cells with these five Cas12a nucleases (Fig. 2g). The maximum cleavage efficiency of CeCas 12 a was about $56 \%$ at site 2 , while BfCas12a presented the modest cleavage activity at VEGFA site 1 and site 2 (Fig. 2g).

Cas12a alone was able to process pre-crRNA and generate mature crRNA $[14,33]$. So it required only a Pol
III promoter to drive transcription and maturation of multiple crRNAs. Thus, we detected whether CeCas12a and BfCas12a retained the activities of multiplex gene editing by using a single crRNA array that contains 4 crRNAs targeting 4 human genes (TRAC, TRBC, B2M, CTLA4). The T7E1 assay showed that CeCas12a achieved impressive cleavage efficiency with about $50 \%$ at CTLA4, $40 \%$ at B2M, 30\% at TRBC, and $23 \%$ at TRAC. In contrast, BfCas12a also achieved about $24 \%$ efficiency at $\mathrm{B} 2 \mathrm{M}, 10 \%$ at TRBC, and $5 \%$ at CTLA4, but no detectable efficiency at TRAC (Fig. 2h).

Taken together, these results further showed that CeCas12a and BfCas12a could be used to introduce insertions or deletions (indels) at target sites in human cells and could also be used for multiplex gene editing.

\section{Cas12a orthologs with different stringencies for recognizing canonical TTTV and non-canonical C- containing PAMs in human cells}

For further checking C-containing PAM tolerance by Cas12a in human cells, As, Lb, and Ce Cas12a nucleases were investigated toward 82 endogenous target sites, with either TTTV, CTTV, TCTV, TTCV, CCTV, TCCV, or CCCV as the potential PAM (Fig. 3a). Target deep sequencing results showed that AsCas12a, LbCas12a, and Ce Cas12a orthologs efficiently modified all 9 target sites with the TTTV PAM (Fig. 3a). When substituting thymidine to cytidine at PAM position $-2,-3$, and -4 , Ce was in a very low activity at those C-containing PAMs (Fig. 3a), and the cleavage ratio of TTCV to TTTV achieved about $40 \%$ for LbCas12a and 25\% for AsCas12a and the cleavage ratio of CTTV to TTTV also achieved about 23\% for LbCas12a and 18\% for AsCas12a (Fig. 3b). In contrast, the CeCas12a cleavage ratios of TTCV to TTTV and CTTV to TTTV were lower (about 20\% and 8\%) (Fig. 3b). These results demonstrated that CeCas12a presents a more rigorous recognition of TTTV PAM in human cells. Thus, this rigorous recognition might have the potential to hinder off-targeting at the C-containing PAM sites. 
a

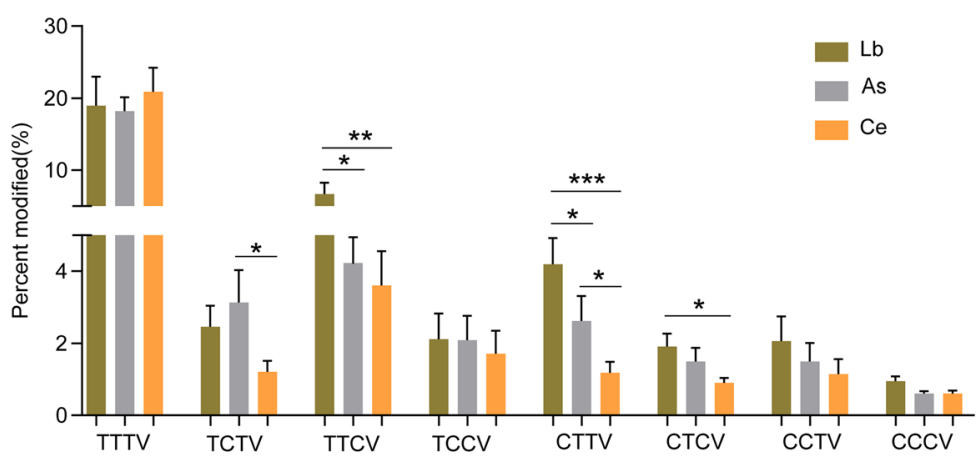

b

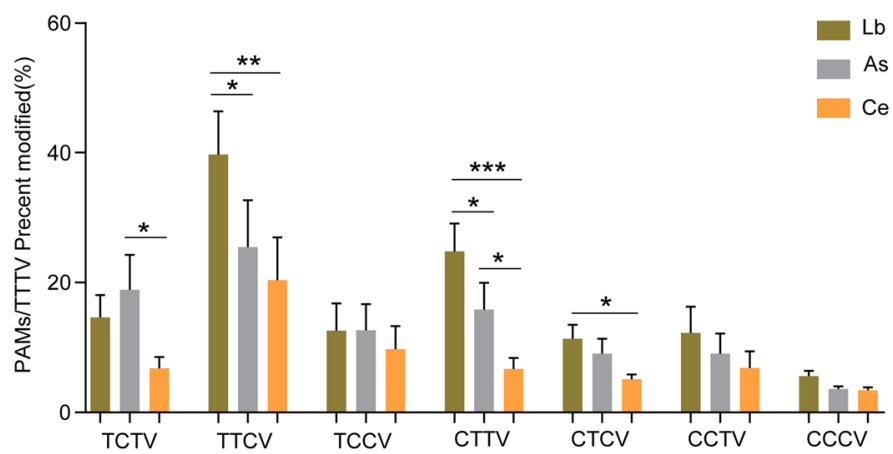

Fig. 3 The stringency of CeCas12a, LbCas12a, and AsCas12a for recognizing canonical TTV and non-canonical C-containing PAMs in human cells. a In vivo cleavage activities of Lb, As, and Ce toward different PAMs. Indel frequencies for 82 endogenous target sites with the different PAMs were measured in mammalian cells by deep sequencing. Percent modified indicates the percentage of reads containing indels compared with the wild-type sequence. $\mathbf{b}$ Stringency for recognizing canonical TTTV and non-canonical C-containing PAMs. The relative percent modified frequency was determined by the ratio of C-containing PAM to TTTV PAM. Data are shown as mean \pm s.e.m. $(n=3) ;{ }^{*} P<0.05,{ }^{* *} P<0.01,{ }^{* * *} P<0.001$ (Mann-Whitney)

Off-targeting was obstructed at C-containing PAM sites of CeCas12a in human cells

AsCas12a and LbCas12a have been reported to introduce gene editing with high specificity in human cells $[17,18]$. To further investigate the fidelity of CeCas12a, DNMT1, $P O L Q, I L 12 A, H B B$, and $B 2 M$ were selected as targets. Forty-one predicted off-target sites with $\mathrm{C}$-containing PAMs and TTTN PAMs were both selected by using CasOFFinder [34] (Additional file 1: Figure S13A, B). After co-transfecting Cas12a plasmids and U6 crRNA fragments for $48 \mathrm{~h}$, the target sites and off-target sites were amplified and went through targeted deep sequencing (Additional file 1: Figure S13A, B). The results confirmed that Cas12a nucleases were highly specific on genomic editing with only 8 sites of DNMT1, POLQ, and IL12A showing low off-target effects (Fig. 4a, c, d; Additional file 1: Figure S13A, B). And CeCas12a showed very low off-target rates as less than $0.05 \%$ in most of cases while maintaining the comparable cleavage efficiency to AsCas12a and LbCas12a (Fig. 4a, d; Additional file 1: Figure S13A, B). In detail, 4/8 predicted off-target sites (OT1, OT3, OT5, OT6) of POLQ were detected for all three Cas12a nucleases (Fig. 4c, d; Additional file 1: Figure S13A, B). For DNMT1 and IL12A, off-target events also happened for all three Cas12a nucleases (Fig. 4c; Additional file 1: Figure S13A, B). Furthermore, 3 off-target sites (POLQ OT1, DNMT1 OT1, IL12A OT1) turned out to have $\mathrm{C}$-containing PAMs, which was TTCA, CTTA, and CTTG, respectively (Fig. 4b; Additional file 1: Figure S13), and CeCas12a had lower indel efficiencies comparable to AsCas12a and LbCas12a (Fig. 4c). For these three $\mathrm{C}$-containing PAM off-target sites of AsCas12a, LbCas12a, and Ce Cas12a orthologs, the off-targeting efficiencies had big differences. The ratio of the off-target (OT1) to on-target exhibited significant differences, and CeCas12a presented the lower ratio, which was also consistent with the in vitro data that more tight stringency for recognizing non-canonical Ccontaining PAMs was revealed for CeCas12a (Fig. 4a-c; Additional file 1: Figure S14). For other off-target sites that mismatches happened in the spacer sequence region, CeCas12a was also revealed to be with less or similar offtarget rates (Fig. 4d). To assess global off-target effects of CeCas12a and compare the genome-wide specificities of CRISPR-Cas nucleases including AsCas12a, enAsCas12aHF1 [35], LbCas12a, spCas9, and BhCas12b v4 [36], we identified five endogenous target sites that contained 


\section{a}

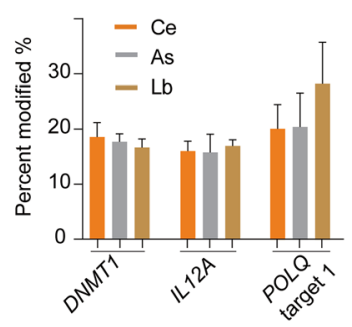

b

PAM $\frac{P O L Q \text { target } 1}{\text { TTTAGGCATGAAT TATAATGCTGTTGG }}$

OT1 TTCAGACATGAATTATAATGCTGTTGG PAM DNMT1 target

TTTGGCTCAGCAGGCACCTGCC TCAGC

OT1 CTTAGCTCAGCAGGCACCTGCCCATGG PAM IL12A target

TTTA $\overline{G G A T G C C A C T A A A A G G G A A A G G G}$

OT1 CTTGAGA TGCCACTAAGAGGGAAAGGG

e Position in Cas 12a/Cas 12b spacer PAM $1,5,10,15,20$, TTTNNNNNNNNNNNNNNNNNNNNNGG Position in SpCas9 spacer
$20,15,10$ PAM

g

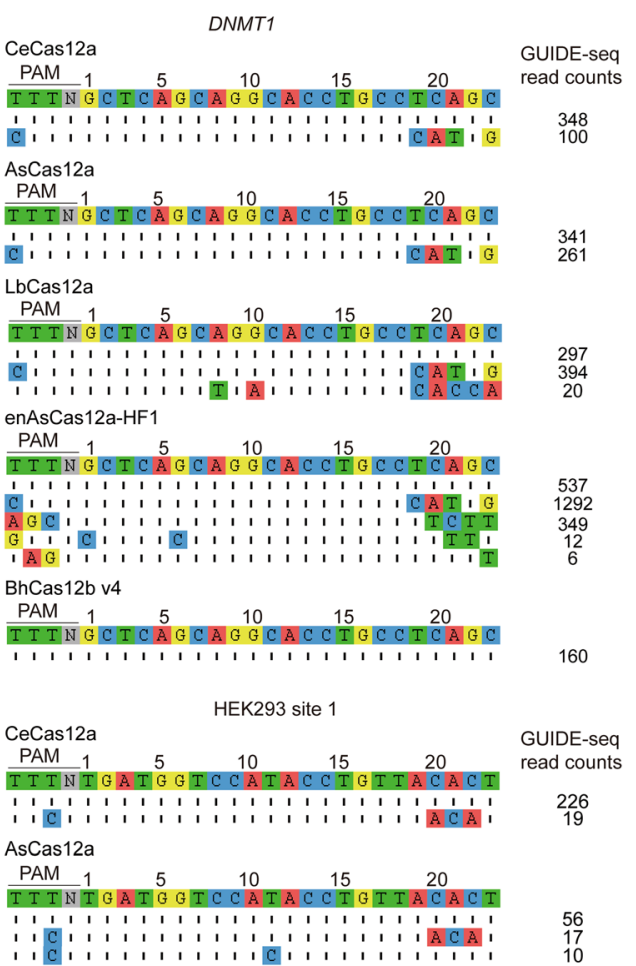

100
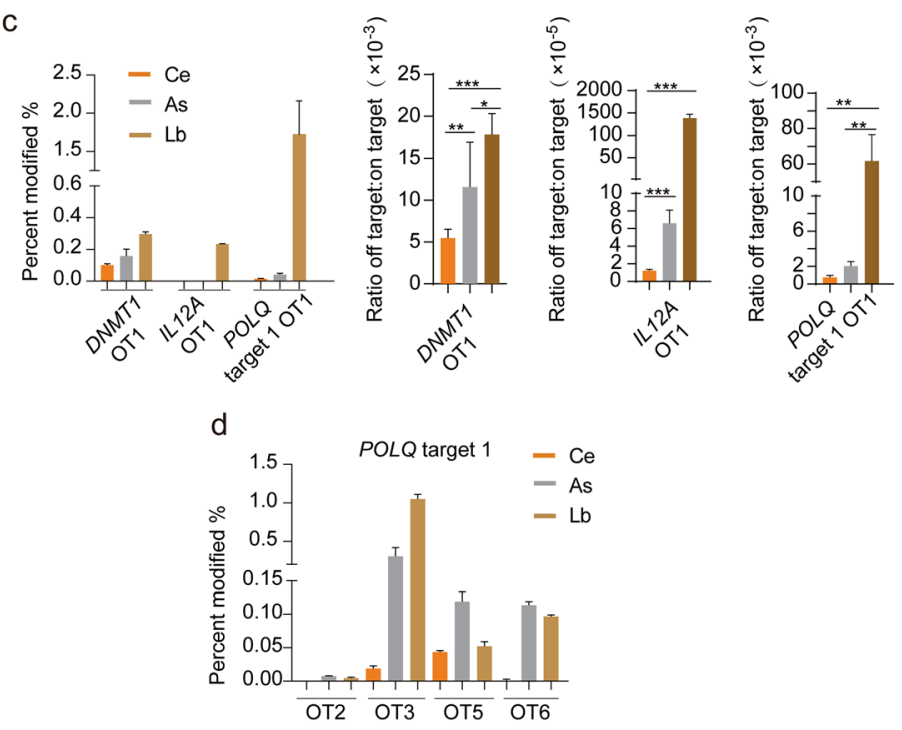

$f$

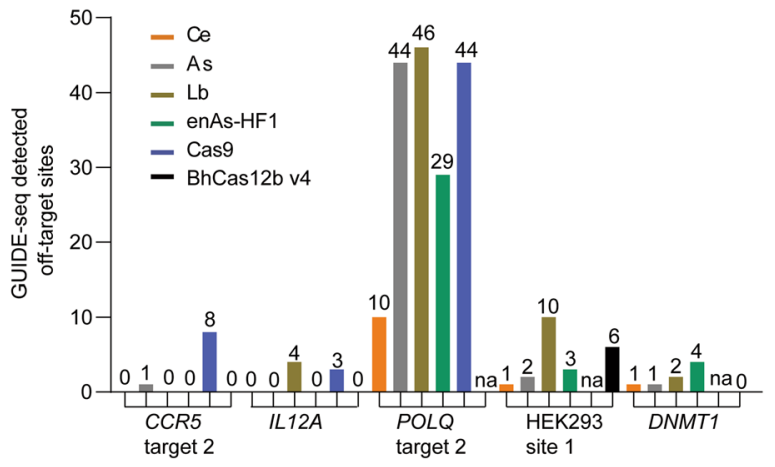

$$
\text { HEK293 site } 1
$$

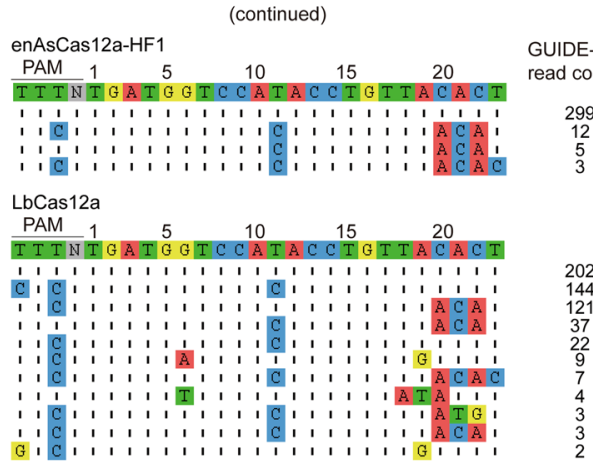

BhCas12b v4

$\frac{\text { PAM }}{\text { T T T N T G T T G T C C A T A C C T GT T A C A C T }}$

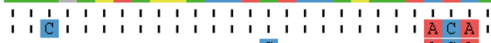

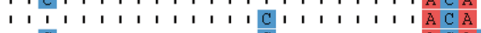

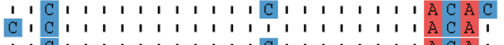

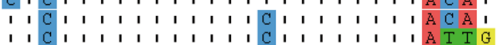

66
87
55
7
5
5
4

Fig. 4 (See legend on next page.) 
(See figure on previous page.)

Fig. 4 Assessment off-target effects of CRISPR-Cas nucleases. a Mean percent modification by Lb, As, and Ce at POLQ, DNMT1, and IL12A on-target sites. Percent modifications were determined by deep sequencing. Bars show mean \pm s.e.m. for $n=3$ transfected cell cultures. Indel mutation frequencies were indicated by the percentage of reads containing indels compared with the wild-type sequence reads. $\mathbf{b}$ Illustration of off-target sites at PAM sequence. Mismatches at PAM sequence and spacer are red. c Mean percent modification at off-target site 1 (OT1) (left), and specificity ratios of LbCas 12a, AsCas12a, and CeCas12a with the POLQ, DNMT1, and IL12A crRNAs, plotted as the ratio of OT1 site activity to on-target activity (right). Data are shown as mean \pm s.e.m. $(n=3)$. ${ }^{*} P<0.05$, ${ }^{*} P<0.01,{ }^{* *} P<0.001$ (Mann-Whitney). $\mathbf{d}$ Mean percent modification by Lb, As, and Ce at off-target sites when targeting POLQ target 1. OT2, OT3, OT5, and OT6 were predicted by Cas-OFFinder, and mismatches are in spacer region (Additional file 1: Figure S13). e Matched target sites for Cas12a, Cas12b, and SpCas9 that share a common protospacer sequence. $\mathbf{f}$ Histograms illustrating the number of GUIDE-seq detected off-target sites for CeCas12a, AsCas12a, LbCas12a, enAsCas12a-HF1, spCas9, and BhCas12b v4. na, not assessed. g Off-target sites for CeCas12a, AsCas12a, LbCas12a, enAsCas12a-HF1, and BhCas12b v4 with DNMT1 and HEK293 site1 crRNAs, determined using GUIDE-seq in HEK293 cells. Mismatched positions are highlighted in color, and GUIDE-seq read counts are shown to the right of the on- or off-target sequences

overlapping target sites (except for HEK293 site 1 and DNMT1) for these CRISPR-Cas nucleases (Fig. 4e). And the genome-wide unbiased identification of doublestranded breaks (DSBs) enabled by sequencing (GUIDEseq) method was used [37]. For two of five target sites evaluated (CCR5 target 2, IL12A), no off-target activities were detected for CeCas12a (Fig. 4f). For other three target sites evaluated (POLQ target 2, HEK293 site 1, and DNMT1), CeCas12a exhibited the similar or reduced number of off-target sites. And off-targeting that happened at PAM sequence was also detected by GUIDE-seq (Fig. 4f, g; Additional file 1: Figure S15) for all tested CRISPR-Cas nucleases, which is constant with the result of targeted deep sequencing.

Taken together, these results indicated the good characters of CeCas12a on the improvement of specific gene editing. Thus, CeCas12a could be another novel prominent Cas12a nuclease with improvements on off-targeting and comparable high cleavage efficiency to AsCas12a and LbCas12a in human cells. Also, the stringency or tolerance on non-canonical PAM sequences might be an important factor when considering off-targeting.

\section{Discussion}

Cas12a was suggested to have low off-target rates in general with high cleave efficiency in mammalian cells and was considered as another kind of promising genomic editing tools. In this work, we report the identification of two novel Cas12a nucleases for genome editing in mammalian cells. One of them, named CeCas12a, was revealed to be more restrictive in vitro on PAM sequences than AsCas12a and LbCas12a. Certain permissions of other amino acids in the TTTV PAM sequence were somehow allowed for AsCas12a and LbCas12a. However, CeCas12a is much more restrictive on this permission. This feature provided the potential of decreasing off-targeting at the PAM region and attracted our interests.

The newly identified Cas12a nucleases were also tested for the efficiency of genome-editing ability in mammalian cells. Comparing to AsCas12a and LbCas12a, CeCas12a was identified to have comparable efficiency in editing multiple gene sites in 293T cells and BfCas12a only had comparable efficiency in limited target sites. The restriction on PAM sequences of CeCas12a was also confirmed in $293 \mathrm{~T}$ cells. Cas12a nucleases such as AsCas12a and LbCas12a have very low off-target rates in general, and in many predicted off-target sites, off-targeting did not show up [17-19, 21]. It could be challenging to find other CRISPR-Cas proteins to have even lower off-target rates than the reported AsCas12a and LbCas12a. However, encouraged by the fact that CeCas12a has a more restricted PAM sequence requirement in vitro, we tested the offtargeting of CeCas12a in human 293T cells. From many off-target sites, several sites showed off-target effects in the PAM region and CeCas12a displayed lower off-target rates than other tested Cas12a. Thus, instead of increasing the scope of PAM to cover more potential target site, another direction of narrowing the scope of PAM for the sake of low off-targeting was indicated. And PAM stringency is not likely to limit the utility of CeCas12a for there is at least one TTTN PAM sequence per 25-bp nucleotides in human genome (Additional file 2: Table S2).

For other off-target sites where off-targeting only happened in the spacer sequence region, CeCas12a also shows similar or reduced off-targeting than other tested CRISPR-Cas nucleases in general. This could be due to the natural properties of CeCas12a, and more studies in the future could help us to understand offtargeting more and shape off-targeting more efficiently. Furthermore, CeCas12a was also confirmed to have the ability to truncate the pre-crRNA to the mature crRNA by itself and performing multigene editing in mammalian cells. Thus, CeCas12a could be another very useful tool in genome editing and disease therapy in the future.

Off-targeting is one of the important issues to hinder the clinical use of genome editing [37-39]. However, off-targeting is complicated and could happen at both the PAM region and the spacer region $[19,21]$. For off- 
targeting at PAM region, the case of CeCas12a suggested that the more specific recognition of PAM sequences in vitro could be followed by the more specific PAM recognition in vivo. Thus, more usable genome-editing tools with low off-target rates could be developed by a novel means of restricting the PAM motif and could be discovered through the methodology of in vitro tested or screened easily at first.

Multiple other different ways have been developed to lower off-targeting by modifying the protein, the crRNA, the delivery, and etc. [40-43]. However, different orthologs of CRISPR-Cas proteins with lower off-target rates are less identified and CeCas12a could be such a good example, which points out that the in vitro property could be a very important guideline to find more useful gene-editing tools. Meanwhile, other ways such as modifying the protein, the crRNA, or the delivery might be easily combined with the newly identified CRISPR-Cas proteins such as CeCas12a. In the future, the combination of different ways to enable higher specificity of CRISPR-Cas nucleases for genome editing could be of great help for reaching the criteria of clinical usage.

\section{Conclusions}

In conclusion, we have shown that the stringency of PAM recognition could be an important factor shaping offtargeting in gene editing which is demonstrated by a novel promising Cas12a ortholog. Two Cas12a orthologs, CeCas12a and BfCas12a, were identified to be active in human cells and able to perform multigene editing. Comparing to well-accepted AsCas12a and LbCas12a, CeCas12a was identified to have comparable efficiency in editing multiple gene sites and BfCas12a only had comparable efficiency in limited target sites. CeCas12a was shown to be more stringent for PAM recognition in vitro and in vivo than AsCas12a and LbCas12a followed by the lower off-targeting at the PAM region, and the offtargeting at other regions was also lower in general. Thus, CeCas12a represents a promising candidate due to its distinctive characteristics, and aiming at the stringency of PAM recognition could be an important clue to find more accurate gene-editing tools in future.

\section{Methods}

\section{Plasmids for Cas12a or crRNA expression}

A list and partial sequences of plasmids used in this study can be found in Table S4. AsCas12a, LbCas12a, and FnCas12a human expression plasmids were purchased from the non-profit plasmid repository Addgene (Addgene plasmids \#69982, \#69988, and \#69976, respectively). CeCas12a and BfCas12a genes were synthesized and cloned into pcDNA3.1(+) for expression in human cells. The open reading frames of the nucleases were amplified by PCR (Phanta MAX Super-Fidelity
DNA Polymerase, Vazyme) from their relevant human expression plasmids and inserted into the $\mathrm{NcoI}$ and XhoI sites of pET28a(+) for E. coli expression. Oligonucleotide duplexes corresponding to Cas12a orthologs and spacer sequences were PCR amplified and ligated into pU6-Fn-crRNA plasmids for U6 promoter-driven expression of crRNAs. The sequences of crRNA used in this study are also listed in Additional file 2: Table S3.

\section{Production of crRNAs}

All crRNAs used in in vitro cleavage experiments were synthesized using the T7 High Yield RNA Transcription kit (Vazyme, TR101-02). ssDNA oligos (Additional file 2: Table S3) corresponding to the reverse complement of the target RNA sequence and a short T7 priming sequence were synthesized from TSING KE and annealed to obtain the template of the transcription reaction. T7 transcription was performed for $16 \mathrm{~h}$, and then RNA was purified using the SanPrep Column PCR Product Purification Kit (Sangon Biotech).

\section{Purification of Cas12a proteins}

The E. coli expression plasmids of the nucleases were transformed in Escherichia coli Rosseta cells. Protein expressions were inducted with $0.3 \mathrm{mM}$ IPTG, $16 \mathrm{~h}$. Cells were resuspended in lysis buffer $(10 \mathrm{mM}$ Tris- $\mathrm{HCl}(\mathrm{pH}$ 8.0), $5 \mathrm{mM} \mathrm{MgCl} 2,200 \mathrm{mM} \mathrm{NaCl}, 5 \mathrm{mM}$ imidazole, $0.1 \%$ Triton-100) and disrupted by Hydraulic Breaker. Cell debris and insoluble particles were removed by centrifugation at $12,000 \mathrm{~g}$ at $4{ }^{\circ} \mathrm{C}$. The lysate was filtered through $0.22-\mu \mathrm{m}$ filters and applied to a nickel column, washed, and then eluted with a gradient of imidazole. Fractions containing protein of the expected size were pooled and applied onto a HiTrap Heparin HP column equilibrated with buffer $\mathrm{L}$ (10 $\mathrm{mM}$ Tris- $\mathrm{HCl}$ (pH 8.0), $5 \mathrm{mM} \mathrm{MgCl}_{2}$, $1 \mathrm{mM}$ DTT, $200 \mathrm{mM} \mathrm{NaCl}$ ). The protein was eluted with a linear gradient of $0-100 \%$ buffer $\mathrm{H}(10 \mathrm{mM}$ Tris- $\mathrm{HCl}$ (pH 8.0), $5 \mathrm{mM} \mathrm{MgCl}_{2}, 1 \mathrm{mM}$ DTT, $1 \mathrm{M} \mathrm{NaCl}$ ). The protein peaks were dialyzed with buffer $\mathrm{L}$ and concentrated. Then, the proteins can be either used directly for biochemical assays or frozen at $-80^{\circ} \mathrm{C}$.

\section{In vitro cleavage assay}

Two hundred fifty-six kinds of linear dsDNA substrates were synthesized consisting of 4 randomized nucleotides upstream of the protospacer with overlap PCR. Cleavage in vitro was performed with purified proteins and corresponding crRNAs at $37^{\circ} \mathrm{C}$ in cleavage buffer $(20 \mathrm{mM}$ HEPES- $\mathrm{NaOH}$ (pH 7.5), $100 \mathrm{mM} \mathrm{KCl,} 2 \mathrm{mM} \mathrm{MgCl} 2,1$ $\mathrm{mM}$ DTT, and $5 \%$ glycerol) for $15 \mathrm{~min}$. The cleavage reaction used $100 \mathrm{nM}$ of synthesized crRNA and $200 \mathrm{ng}$ of target DNA. Reactions were terminated with Protease K at $58{ }^{\circ} \mathrm{C}$ for $30 \mathrm{~min}$ and were run on $2 \%$ agarose gels. 


\section{In vitro PAM identification assay}

PAM library included 256 kinds of linear dsDNA substrates consisting of 4 randomized nucleotides upstream of $23 \mathrm{nt}$ protospacer, and dsDNA substrates were mixed with each other at the same concentration. Each in vitro cleavage reaction consisted of $100 \mathrm{ng}$ PAM library, 100 nM Cas12a RNP complex, $1 \mu \mathrm{L} 10 \times$ CutSmart buffer (NEB), and water for a total volume of $10 \mu \mathrm{L}$. Each cleavage reaction was incubated at $37^{\circ} \mathrm{C}$ and quenched at $0 \mathrm{~min}, 1 \mathrm{~min}, 2 \mathrm{~min}, 4 \mathrm{~min}, 6 \mathrm{~min}, 8 \mathrm{~min}, 10 \mathrm{~min}, 16$ $\mathrm{min}$, and $32 \mathrm{~min}$. Reactions were purified with magnetic beads (VAHTS DNA Clean Beads, Vazyme), and $10 \mathrm{ng}$ purified dsDNA was amplified with PCR over 20 total cycles using custom primers containing Illumina adaptors. The unmodified library amplicon was used as a negative control to determine initial PAM representation in the libraries. Purified PCR products were quantified followed by sequencing with a 75-cycle NextSeq kit (Illumina), and PAM regions were extracted, counted, and normalized to total reads for each sample.

\section{Analysis of PAM cleavage kinetics}

Sequencing reads were filtered by Phred quality ( $\geq 30$ for all of 256 kinds of PAM bases) and were analyzed using a custom Python script to estimate cleavage rates on each PAM for each of nuclease [35]. For each cleavage reaction, we calculated the depletion ratio (normalized read count in cleavage reaction)/(normalization read count in negative control) and then divided by the median depletion ratio of all VRRT sequences, which were not cleaved by Cas12a nucleases [19]. Each PAM (256 total) deletion ratio for each Cas12a nuclease over time fit to non-linear least squares to an exponential decay model $\left(y(t)=A e^{-k t}\right.$, where $y(t)$ is the normalized PAM count, $t$ is the time (minutes), $A$ is a constant, and $k$ is the rate constant).

\section{Cell culture and transfection}

HEK293T cells were cultured at $37{ }^{\circ} \mathrm{C}$ with $5 \% \mathrm{CO}_{2}$ in DMEM supplemented with $10 \%$ heat-inactivated fetal bovine serum and $1 \%$ penicillin/streptomycin (all cell culture products were obtained from Gibco). Cells were seeded 1 day prior to transfection in 24- or 96-well plates at a density of approximately $1.2 \times 105$ cells per 24 -well or $2.4 \times 104$ cells per 96-well and transfected at 50-80\% confluency using Hieff Trans Liposomal Transfection Reagent (CAT:40802ES03, Yeasen, Shanghai). For indel analysis or EGFP disruption analysis in HEK293T cells, a total of $500 \mathrm{ng}$ of Cas12a plasmid plus 200 ng crRNA plasmid was delivered per 24-well or 100 ng Cas12a plus 50 ng crRNA plasmid per 96-well.

\section{EGFP disruption assay}

Human 293T cells were transfected with Cas12a expression plasmid, EGFP expression plasmid, and crRNA expression plasmid, or an U6 promoter-driven empty plasmid for the substitution of crRNA expression plasmid as a negative control. Forty-eight hours post-transfection, cells were analyzed on the CytoFLEX (Beckman Coulter). For each sample, transfections and flow cytometry measurements were performed in triplicate.

\section{T7E1 assays}

Cells were collected after $48 \mathrm{~h}$ post-transfection for genomic DNA extraction using Animal Tissue Direct PCR Kit (CAT:10180ES70, Yeasen, Shanghai). The genomic region flanking the Cas12a targeting site of each gene was PCR amplified, and products were purified using SanPrep Column PCR Product Purification Kits (Sangon Biotech) following the manufacturer's protocol. A total of $\sim 200 \mathrm{ng}$ purified PCR amplicons were denatured, reannealed, and digested with T7E1 (Vazyme). The reaction mixtures were run on $2 \%$ agarose gels after incubation for $20 \mathrm{~min}$ at $37^{\circ} \mathrm{C}$. The gels were imaged with ChemiDocTM XRS+ and analyzed according to strip intensities. Indel percentage was determined by the formula: $100 \times(1-\operatorname{sqrt}(b+c) /$ $(a+b+c))$, where $a$ is the integrated intensity of the undigested PCR product and $b$ and $c$ are the integrated intensities of the cleavage product.

\section{Targeted deep sequencing assays}

Off-targets were predicted using the online website CasOFFinder (http://www.rgenome.net/cas-offinder/), and the relevant primers were designed. Each predicted offtarget fragment is approximately $250 \mathrm{bp}$. These primers were used for PCR amplification amplified using corresponding genomic DNAs as templates. The purified PCR products were quantified by Infinite 200 (TECAN). Each $300 \mathrm{ng}$ predicted off-target fragment was mixed and sent to Novogene for deep sequencing, and off-target analysis was performed according to the sequencing results. All of the above experiments were performed in three biological replicates.

\section{GUIDE-seq}

GUIDE-seq experiments were performed as previously described [37]. Briefly, $100 \mathrm{pmol}$ of the double-stranded oligodeoxynucleotide (dsODN) GUIDE-seq tag was transfected into $293 \mathrm{~T}$ cells with CRISPR-Cas nuclease protein and guide RNA expression plasmids. On-target modification and GUIDE-seq tag integration percentages were assessed by using T7E1 assays (as described above) and restriction-fragment length polymorphisms (RFLP) assays, respectively. GUIDE-seq libraries were sequenced using an Illumina sequencer, and data was analyzed using guideseq v1.1 as described previously [37, 44]. 


\section{Statistics}

Statistical significance was calculated using MannWhitney tests using GraphPad Prism version 8.0. The error bars in all figures show standard error of the mean $(n=3) . P$ values are reported using GraphPad style: not significant (ns), $P>0.05 ; *, P<0.05 ;{ }^{* * *}, P<0.01$; ***; $P<$ 0.001 .

\section{Supplementary information}

Supplementary information accompanies this paper at https://doi.org/10. 1186/s13059-020-01989-2.

\section{Additional file 1: Figure S1. Diagram of Cas12a loci. Figure S2.} Expression of Cas12a orthologs in E. coli cells. Figure S3. Substrates synthesis and cleavage assay. Figure S4. Extended gel image of Fig. 1a. Figure S5. DNA cleavage activity of BhCas12a in vitro. Figure S6. DNA cleavage activity of CsbCas12a in vitro. Figure S7. DNA cleavage activity of PrCas12a in vitro. Figure S8. Quantification of time-course in vitro cleavage activities of Cas12a orthologs. Figure \$9. Multiple sequence alignment of Cas12a RuvC domains. Figure S10. Efficiencies of EGFP disruption mediated by As, Lb and Fn. Figure S11. Mutations induced by CeCas12a and BfCas12a. Figure S12. Gel images of Fig. 2f. Assessment of gene editing efficiencies with CeCas12a. Figure S13. Off-target effects of Cas12a-mediated gene editing in human cells. Figure S14. Off-target effects of Cas12a-mediated gene editing at C-containing PAM sites in human cells. Figure S15. Specificity analysis of matched CRISPR-Cas nuclease targets

Additional file 2: Table S1. crRNA array locus of different Cas12a orthologs. Table S2: TTTN PAM distribution. Table S3. List of sequences used in study. Table S4. Oligonucleotides (oligos) for Cas12a gene synthesis.

Additional file 3. Sequencing data for PAM identification and PAM cleavage kinetics analysis.

Additional file 4. Review history.

\section{Acknowledgements}

We thank all the members of our laboratory for the fruitful discussions and support.

\section{Peer review information}

Yixin Yao was the primary editor of this article and managed its editorial process and peer review in collaboration with the rest of the editorial team.

\section{Review history}

The review history is available as Additional file 4.

\section{Authors' contributions}

Peng Chen, Jin Zhou, and Yibin Wan contributed equally to this work. Lei Yin and Peng Chen conceptualized the study, designed the experiments, and wrote the manuscript. Peng Chen performed the experiments and analyzed the data. Jin Zhou and Yibin Wan established pET-28-Cas12a plasmids and purified Cas12a proteins and assisted with cleavage experiments in vitro. Huan Liu assisted with the primer design and cleavage experiments in vivo. Yongzheng Li processed the sequencing data and assisted with the analysis. Zhaoxin Liu, Hongjian Wang, Jun Lei, and Kai Zhao assisted with the preparation of 256 kinds of substrates. Yibin Wan, Huan Liu, and Yiliang Zhang assisted with the preparation of PCR products for deep sequencing. Xinghua Zhang and Yan Wang assisted with the modification of this paper. Lei Yin supervised the study. The authors reviewed and approved the final manuscript.

\section{Funding}

This work was supported by the National Natural Science Foundation of China (31870728 and 31470738), the National Basic Research Program of China (2014CB910103), and the Science Foundation of Wuhan University (2042016kf0169).

\section{Availability of data and materials}

The sequences of expression cassettes used in this paper are listed in Additional file 2. Raw sequencing reads are available at the Gene Expression Omnibus under accession GSE146420 [45]. All materials described in this study are freely available upon request.

\section{Ethics approval and consent to participate}

Not applicable

\section{Competing interests}

The authors declare that they have no competing interests.

\section{Author details}

${ }^{1}$ State Key Laboratory of Virology, Hubei Key Laboratory of Cell Homeostasis, Department of Biochemistry and Molecular Biology, College of Life Sciences, Wuhan University, Wuhan, China. ${ }^{2}$ Department of Clinical Oncology, Renmin Hospital of Wuhan University, Wuhan 430060, China. ${ }^{3}$ Hubei Key Laboratory of Cell Homeostasis, College of Life Sciences, Wuhan University, Wuhan 430072, Hubei, People's Republic of China. ${ }^{4}$ College of Life Sciences, the Institute for Advanced Studies, State Key Laboratory of Virology, Hubei Key Laboratory of Cell Homeostasis, Wuhan University, Wuhan 430072, China.

Received: 30 October 2019 Accepted: 6 March 2020

Published online: 25 March 2020

\section{References}

1. Chen B, Gilbert LA, Cimini BA, Schnitzbauer J, Zhang W, Li GW, Park J, Blackburn EH, Weissman JS, Qi LS, Huang B. Dynamic imaging of genomic loci in living human cells by an optimized CRISPR/Cas system. Cell. 2013; 155:1479-91.

2. Ma H, Naseri A, Reyes-Gutierrez P, Wolfe SA, Zhang S, Pederson T. Multicolor CRISPR labeling of chromosomal loci in human cells. Proc Natl Acad Sci U S A. 2015;112:3002-7.

3. Rees HA, Liu DR. Base editing: precision chemistry on the genome and transcriptome of living cells. Nat Rev Genet. 2018:19:770-88.

4. Cong L, Ran FA, Cox D, Lin S, Barretto R, Habib N, Hsu PD, Wu X, Jiang W, Marraffini LA, Zhang F. Multiplex genome engineering using CRISPR/Cas systems. Science. 2013;339:819-23.

5. Gilbert LA, Larson MH, Morsut L, Liu Z, Brar GA, Torres SE, Stern-Ginossar N, Brandman O, Whitehead EH, Doudna JA, Lim WA, et al. CRISPR-mediated modular RNA-guided regulation of transcription in eukaryotes. Cell. 2013; 154:442-51.

6. Qi LS, Larson MH, Gilbert LA, Doudna JA, Weissman JS, Arkin AP, Lim WA. Repurposing CRISPR as an RNA-guided platform for sequence-specific control of gene expression. Cell. 2013;152:1173-83.

7. Mali P, Yang L, Esvelt KM, Aach J, Guell M, JE DC, Norville JE, Church GM RNA-guided human genome engineering via Cas9. Science. 2013;339:823-6.

8. Konermann S, Brigham MD, Trevino AE, Joung J, Abudayyeh $\mathrm{OO}$, Barcena C, Hsu PD, Habib N, Gootenberg JS, Nishimasu H, et al. Genome-scale transcriptional activation by an engineered CRISPR-Cas9 complex. Nature. 2015;517:583-8

9. Cho SW, Kim S, Kim JM, Kim JS. Targeted genome engineering in human cells with the Cas9 RNA-guided endonuclease. Nat Biotechnol. 2013;31:230-2.

10. Jiang W, Bikard D, Cox D, Zhang F, Marraffini LA. RNA-guided editing of bacterial genomes using CRISPR-Cas systems. Nat Biotechnol. 2013;31:233-9.

11. Ran FA, Cong L, Yan WX, Scott DA, Gootenberg JS, Kriz AJ, Zetsche B, Shalem O, Wu X, Makarova KS, et al. In vivo genome editing using Staphylococcus aureus Cas9. Nature. 2015;520:186-91.

12. Esvelt KM, Mali P, Braff Jl, Moosburner M, Yaung SJ, Church GM. Orthogonal Cas9 proteins for RNA-guided gene regulation and editing. Nat Methods. 2013;10:1116-21.

13. Hou Z, Zhang Y, Propson NE, Howden SE, Chu LF, Sontheimer EJ, Thomson JA. Efficient genome engineering in human pluripotent stem cells using Cas 9 from Neisseria meningitidis. Proc Natl Acad Sci U S A. 2013;110:15644-9.

14. Zetsche B, Gootenberg JS, Abudayyeh OO, Slaymaker IM, Makarova KS, Essletzbichler P, Volz SE, Joung J, Van Der Oost J, Regev A, et al. Cpf1 is a single RNA-guided endonuclease of a class 2 CRISPR-Cas system. Cell. 2015; 163:759-71.

15. Tu M, Lin L, Cheng Y, He X, Sun H, Xie H, Fu J, Liu C, Li J, Chen D, et al. A 'new lease of life': FnCpf1 possesses DNA cleavage activity for genome editing in human cells. Nucleic Acids Res. 2017:45:11295-304. 
16. Tóth E, Czene BC, Kulcsár PI, Krausz SL, Tálas A, Nyeste A, Varga É, Huszár K, Weinhardt N, Ligeti Z, et al. Mb- and FnCpf1 nucleases are active in mammalian cells: activities and PAM preferences of four wild-type Cpf1 nucleases and of their altered PAM specificity variants. Nucleic Acids Res. 2018;46:10272-85.

17. Kleinstiver BP, Tsai SQ, Prew MS, Nguyen NT, Welch MM, Lopez JM, ZR MC Aryee MJ, Joung JK. Genome-wide specificities of CRISPR-Cas Cpf1 nucleases in human cells. Nat Biotechnol. 2016;34:869-74.

18. Kim D, Kim J, Hur JK, Been KW, Yoon SH, Kim JS. Genome-wide analysis reveals specificities of $\mathrm{Cpf1}$ endonucleases in human cells. Nat Biotechnol. 2016;34:863-8

19. Gao L, http://orcid.org I-O, Cox DB, Yan WX, Manteiga JC, Schneider MW, Yamano T, Nishimasu $H$, Nureki $O$, et al. Engineered Cpf1 variants with altered PAM specificities. Nat Biotechnol. 2017;35:789-92.

20. Zetsche B, Heidenreich M, Mohanraju P, Fedorova I, Kneppers J, EM DG, Winblad N, Choudhury SR, Abudayyeh OO, Gootenberg JS, et al. Multiplex gene editing by CRISPR-Cpf1 using a single crRNA array. Nat Biotechnol. 2017;35:31-4.

21. Kocak DD, Josephs EA, Bhandarkar V, Adkar SS, Kwon JB, Gersbach CA Increasing the specificity of CRISPR systems with engineered RNA secondary structures. Nat Biotechnol. 2019:37:657-66.

22. Zetsche B, Strecker J, Abudayyeh OO, Gootenberg JS, Scott DA, Zhang F. A survey of genome editing activity for 16 Cas12a orthologs. Keio J Med. 2019;14:2019-0009. https://doi.org/10.2302/kjm.2019-0009-OA LID.

23. Yamano T, Zetsche B, Ishitani R, Zhang F, Nishimasu H, Nureki O. Structural basis for the canonical and non-canonical PAM recognition by CRISPR-Cpf1. Mol Cell. 2017:67:633-45

24. Tang X, Lowder LG, Zhang T, Malzahn AA, Zheng X, Voytas DF, Zhong Z, Chen Y, Ren $Q$, et al. A CRISPR-Cpf1 system for efficient genome editing and transcriptional repression in plants. Nat Plants. 2017;3:18.

25. Kim HK, Song M, Lee J, Menon AV, Jung S, Kang YM, Choi JW, Woo E, Koh $\mathrm{HC}$, et al. In vivo high-throughput profiling of CRISPR-Cpf1 activity. Nat Methods. 2017;14:153-9.

26. Dai X, Park JJ, Du Y, Kim HR, Wang G, Errami Y, Chen S, Id O. One-step generation of modular CAR-T cells with AAV-Cpf1. Nat Methods. 2019;16: 247-54.

27. Reyon D, Tsai SQ, Khayter C, Foden JA, Sander JD, Joung JK. FLASH assembly of TALENs for high-throughput genome editing. Nat Biotechnol. 2012;30:460-5

28. Fu Y, Foden JA, Khayter C, Maeder ML, Reyon D, Joung JK, Sander JD. Highfrequency off-target mutagenesis induced by CRISPR-Cas nucleases in human cells. Nat Biotechnol. 2013;31:822-6.

29. Kleinstiver BP, Pattanayak V, Prew MS, Tsai SQ, Nguyen NT, Zheng Z, Joung JK. High-fidelity CRISPR-Cas9 nucleases with no detectable genome-wide off-target effects. Nature. 2016;529:490-5.

30. Yamano T, Nishimasu H, Zetsche B, Hirano H, Slaymaker IM, Li Y, Fedorova I, Nakane T, Makarova KS, Koonin EV, et al. Crystal structure of Cpf1 in complex with guide RNA and target DNA. Cell. 2016;165:949-62.

31. Teng F, Li J, Cui T, Xu K, Guo L, Gao Q, Feng G, Chen C, Han D, Zhou Q, Li W. Enhanced mammalian genome editing by new Cas12a orthologs with optimized crRNA scaffolds. Genome Biol. 2019;20:019-1620.

32. Li B, Zhao W, Luo X, Zhang X, Li C, Zeng C, Dong Y. Engineering CRISPRCpf1 crRNAs and mRNAs to maximize genome editing efficiency. Nat Biomed Eng. 2017;1:017-0066. https://doi.org/10.1038/s41551-017-0066 LID - 0066 [pii] LID.

33. Fonfara I, Richter $\mathrm{H}$, Bratovič M, Le Rhun A, Charpentier E. The CRISPRassociated DNA-cleaving enzyme Cpf1 also processes precursor CRISPR RNA. Nature. 2016;532:517-21.

34. Bae S, Park J, Kim JS. Cas-OFFinder: a fast and versatile algorithm that searches for potential off-target sites of Cas9 RNA-guided endonucleases. Bioinformatics. 2014;30:1473-5.

35. Kleinstiver BP, Sousa AA, Walton RT, Tak YE, Hsu JY, Clement K, Welch MM, Horng JE, et al. Engineered CRISPR-Cas12a variants with increased activities and improved targeting ranges for gene, epigenetic and base editing. Nat Biotechnol. 2019;37:276-82.

36. Strecker J, Jones S, Koopal B, Schmid-Burgk J, Zetsche B, Gao L, Makarova $\mathrm{KS}$, Koonin EV, et al. Engineering of CRISPR-Cas12b for human genome editing. Nat Commun. 2019;10:018-08224.

37. Tsai SQ, Zheng Z, Nguyen NT, Liebers M, Topkar W, Thapar V, Wyvekens N, Khayter C, lafrate AJ, Le LP, et al. GUIDE-seg enables genome-wide profiling of off-target cleavage by CRISPR-Cas nucleases. Nat Biotechnol. 2015;33:187-97.
38. Pattanayak V, Lin S, Guilinger JP, Ma E, Doudna JA, Liu DR. High-throughput profiling of off-target DNA cleavage reveals RNA-programmed Cas9 nuclease specificity. Nat Biotechnol. 2013;31:839-43.

39. Kuscu C, Arslan S, Singh R, Thorpe J, Adli M. Genome-wide analysis reveals characteristics of off-target sites bound by the Cas9 endonuclease. Nat Biotechnol. 2014;32:677-83.

40. Slaymaker IM, Gao L, Zetsche B, Scott DA, Yan WX, Zhang F. Rationally engineered Cas9 nucleases with improved specificity. Science. 2016;351:84-8.

41. Yin H, Song CQ, Suresh S, Kwan SY, Wu Q, Walsh S, Ding J, Bogorad RL, Zhu LJ, Wolfe SA, et al. Partial DNA-guided Cas9 enables genome editing with reduced off-target activity. Nat Chem Biol. 2018;14:311-6.

42. Fu Y, Sander JD, Reyon D, Cascio VM, Joung JK. Improving CRISPR-Cas nuclease specificity using truncated guide RNAs. Nat Biotechnol. 2014;32: 279-84.

43. Ran FA, Hsu PD, Lin CY, Gootenberg JS, Konermann S, Trevino AE, Scott DA, Inoue A, Matoba S, Zhang Y, Zhang F. Double nicking by RNA-guided CRISPR Cas9 for enhanced genome editing specificity. Cell. 2013;154:1380-9.

44. Kleinstiver BP, Prew MS, Tsai SQ, Topkar W, Nguyen NT, Zheng Z, Gonzales AP, Li Z, Peterson RT, Yeh JR, et al. Engineered CRISPR-Cas9 nucleases with altered PAM specificities. Nature. 2015;523:481-5.

45. Peng Chen, Jin Zhou, Yibin Wan, Huan Liu, Yongzheng Li, Zhaoxin Liu, Hongjian Wang, Jun Lei, Kai Zhao, Yiliang Zhang, Yan Wang, Xinghua Zhang, Lei Yin. A Cas12a ortholog with stringent PAM recognition followed by low off-target editing rates for genome editing. Datasets. Gene Expression Omnibus. https://www.ncbi.nlm.nih.gov/geo/query/acc.cgi?acc= GSE146420. Accessed 6 Mar 2020

\section{Publisher's Note}

Springer Nature remains neutral with regard to jurisdictional claims in published maps and institutional affiliations.

Ready to submit your research? Choose BMC and benefit from:

- fast, convenient online submission

- thorough peer review by experienced researchers in your field

- rapid publication on acceptance

- support for research data, including large and complex data types

- gold Open Access which fosters wider collaboration and increased citations

- maximum visibility for your research: over $100 \mathrm{M}$ website views per year

At BMC, research is always in progress.

Learn more biomedcentral.com/submissions 